\title{
Ablation of MCMIO using CRISPR/Cas9 restrains the growth and migration of esophageal squamous cell carcinoma cells through inhibition of Akt
} signaling

Jie Yan, ${ }^{1,2}$ Pan Du, ${ }^{3}$ Yongxu Jia, ${ }^{2}$ Zhiwei Chang, ${ }^{2}$ Silin Gan, ${ }^{4}$ Xiaohan Xu, ${ }^{5}$ Yaohe Wang, ${ }^{3}$ Yanru Qin, ${ }^{2}$ Quancheng Kan'

'Department of Gastroenterology, The First Affiliated Hospital of Zhengzhou University, Zhengzhou, China; ${ }^{2}$ Department of Oncology, The First Affiliated Hospital of Zhengzhou University, Zhengzhou, China;

${ }^{3}$ National Center for International Research in Cell and Gene Therapy, Sino-British Research Centre for Molecular Oncology, Zhengzhou University, Zhengzhou, China; ${ }^{4}$ Department of Hematology, The First Affiliated Hospital of Zhen University, Zhengzhou, China; ${ }^{5}$ Department of Anesthesig sy, The First Affiliated Hospital Chengzhou University, Zhengzhor hina

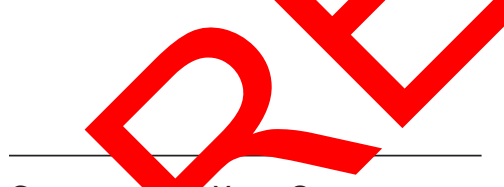

Correspond Yanru Qin Department of rology, The First Affiliated Hospital Zhengzhou University, No I East-Jian She Road, Zhengzhou 45002, China

Tel +86037I6627 II57

Email yanruqin@।63.com

Quancheng Kan

Department of Gastroenterology, The First Affiliated Hospital of Zhengzhou University, No I East-Jian She Road, Zhengzhou 45002, China Tel +8603716 6293114 Email kanquancheng2017@I63.com
This article was published in the following Dove Press journal: OncoTargets and Therapy

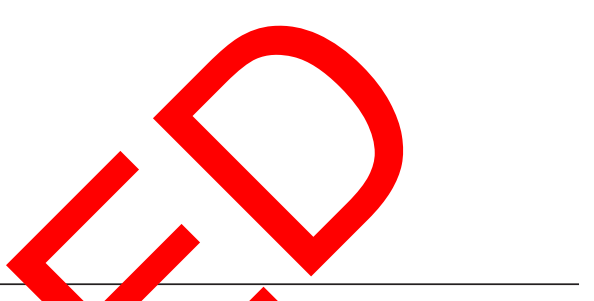

Introduction: Minichromosome main rance 10 $4 \mathrm{CM} 10$ is deregulated in several malignancies including cervical cancer a othelial caro However, the expression and biologic role of MCM10 in esophaged squan cell carcinoma (ESCC) is still unknown.

Methods: In this study, we per immonoh hemistry and real-time polymerase chain reaction (PCR) analysis to amine the expression of MCM10 in ESCC and adjacent normal esophageal tissues. The ass iations of MC 10 expression with clinicopathologic parameters of ESCC were analyzed. Ab ion of MCN 0 through the CRISPR/Cas9 technology was con-

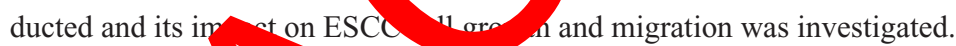

Results: The ml A andein expression levels of MCM10 were significantly greater in ESCC than in norm tiss s(P o $(P)$. The expression of MCM10 was significantly associated with agnosi $=0.033$ ), but not with gender, differentiation grade, invasion status, or ty d-node netastas (TNM) stage. Knockout of MCM10 significantly suppressed the proation conacity of EC109 ESCC cells, compared to control cells ooring wild-type MCM10. Mechanistically, MCM10 depletion markedly reduced the phospho ation of Akt. Overexpression of constitutively active Akt significantly restored the aggressive prenotype of MCM10-null EC109 cells.

nclusion: In conclusion, these results suggest that MCM10 acts as an oncogene in ESCC throsh activation of Akt signaling and represents a promising therapeutic target for this malignancy.

Keywords: esophageal cancer, growth, migration, minichromosome maintenance proteins

\section{Introduction}

Esophageal cancer is one of the most frequently diagnosed cancers worldwide. ${ }^{1}$ In Eastern Asian countries including China, esophageal squamous cell carcinoma (ESCC) is the major histologic type of esophageal cancer. ${ }^{2}$ Despite advances in the prevention and treatment of esophageal cancer, the prognosis of this malignancy is still poor. It is estimated that the overall 5-year survival rate for ESCC is $<40 \%{ }^{3,4}$ Identification of the mechanisms underlying the growth and progression of ESCC is of importance in combating this disease.

Minichromosome maintenance (MCM) proteins are highly conserved in eukaryotes and play an essential role in DNA replication initiation and elongation. ${ }^{5} \mathrm{MCM} 2-7$ proteins are related to each other and form the hexameric complex as a key component of the prereplication complex, contributing to DNA unwinding. MCM1, 
MCM8, and MCM10 are distinct from MCM2-7 proteins, but also participate in DNA synthesis. ${ }^{6}$ Despite the lack of enzymatic domains, MCM10 can interact with replication factors including the MCM2-7 complex and thus coordinate DNA replication. ${ }^{7}$

There is growing evidence for the implication of MCM proteins in cancer progression. ${ }^{8-10}$ For example, high expression of MCM3 is associated with epithelial-mesenchymal transition and invasion in prostate cancer. ${ }^{8}$ Likewise, upregulation of MCM7 is linked to aggressive parameters and poor prognosis in pituitary adenoma. ${ }^{9}$ Blocking MCM2 activity has been reported to enhance DNA damage-induced apoptosis in breast cancer cells. ${ }^{10}$ A previous study has shown that MCM10 is significantly overexpressed in cervical cancer specimens relative to normal cervical tissues. ${ }^{11}$ Another study demonstrated that MCM10 overexpression is associated with advanced stage, nodal metastasis, and vascular invasion, and predicts adverse prognosis in urothelial carcinoma. ${ }^{12}$ However, the expression and biologic role of MCM10 in esophageal cancer is still unclear.

In this study, we examined the expression of MCM10 in 64 pairs of ESCC samples and adjacent normal esophageal tissues and evaluated the relationship between MCM10 expression and clinicopathologic features of ESCC. Moover, the consequence of deletion of MCM10 through th CRISPR/Cas9 technology in ESCC cells was de ined.

\section{Materials and methods}

\section{Tissue specimens}

We collected 64 pairs of formalin paraffin- bedded and 56 pairs of fresh cancerous a norm sophageal missues from ESCC patients who un rwent curativo rgery between 2003 and 2004 at Linzho eople' 'Yospital (Linzhou, China). No patient received ra the or chemotherapy before operation. All car ere plogica confirmed as ESCC. Among the $\mathrm{p}$ ents, $\mathrm{t}$ re wer romen and $36 \mathrm{men}$, with a median of 60 (range 40-80 years). Lymph-node metastasis wa ected in 38 cases. The study was approved by the Ethical iew Board of Zhengzhou University (Zhengzhou, China). Nritten informed consent was obtained from each patient for the use of their tissue in research.

\section{Immunohistochemical staining for MCMIO} Immunohistochemical analysis of MCM10 was performed following a standard protocol. In brief, tissue sections were deparaffinized, rehydrated, and incubated with $3 \% \mathrm{H}_{2} \mathrm{O}_{2}$ to eliminate endogenous peroxidase activity. After blocking with 5\% normal goat serum, sections were incubated with rabbit anti-MCM10 polyclonal antibody (1:500 dilution; Atlas, Stockholm, Sweden) at $4{ }^{\circ} \mathrm{C}$ overnight, followed by biotinylated goat anti-rabbit immunoglobulin for $30 \mathrm{~min}$ at room temperature. After washing, sections were incubated with 3,3'-diaminobenzidine used as a chromogen and counterstained with hematoxylin. Negative controls were included using nonimmune serum.

The evaluation of immunohistochemical results was done in a blind manner by two independent pathologists. A final staining score was calculated by multiplying the scores for staining intensity ( 0 , negative; 1 , weak; 2 , intermediate; 3 , strong) and percentage of positive $<25 \%$ positive cells; $1,25 \%-50 \%$ positive ce $, 2,50 \%-\lambda$ positive cells; $3,>75 \%$ positive cells). Sam ${ }_{\mathrm{p}}$ were defin das high MCM10 expression when thal sco were -9 .

\section{Real-time}

Fresh tissue sa les were med frozen in liquid nitrogen aft surs 1 resection 1 otal RNA from tissues and cells was extrad using TRIzol reagent (Thermo Fishe scientific, Walthà, MA, USA). cDNA was synthe zed using HiScript Q Select RT SuperMix for qua itative PCI and random hexamers from Vazyme Biote Nani g, China). Quantitative real-time PCR carried out with the SYBR Green PCR Master Mix Zyn Biotech). PCR primers are as follows: MCM10 forward, 5'-CACAGAAATGAACAAGAA-3' and MCM10 pverse, 5'-AATAAGAACAAGGACACA-3'; GAPDH forward, 5'-GGAGCGAGATCCCTCCAAAAT-3' and GAPDH reverse, 5'-GGCTGTTGTCATACTTCTCATGG-3'. GAPDH was used as a loading control. The relative expression of MCM10 was calculated using the $2^{-\Delta \Delta \mathrm{Ct}}$ method. ${ }^{13}$

\section{Reverse transcription PCR (RT-PCR) analysis}

Total RNA was extracted and reverse-transcribed to cDNA, as described above. PCR amplification was conducted using the same primers as used in real-time PCR. PCR products were subjected to $1 \%$ agarose gel electrophoresis and stained with ethidium bromide.

\section{Cell culture}

K520, K510, K410, K30, K180, HKESC1, EC7906, EC18, and EC109 ESCC cells were kindly provided by Professor Srivastava (University of Hong Kong). Cells were maintained in Dulbecco's Modified Eagle's Medium (DMEM) (Invitrogen) supplemented with 10\% fetal bovine serum (FBS; Thermo Fisher Scientific). The usage of the cell 
lines was approved by the Ethical Review Board of Zhengzhou University.

\section{Plasmid construction}

For generation of MCM10-targeting single-guide RNAs (sgRNAs), three pairs of oligonucleotides were designed with the sequences as follows: sgRNA-1 forward, 5'-ACCGGAGGCTGATGATGGAGAAAC-3' and sgRNA-1 reverse, 5'-AAACGTTTCTCCATCATCAGCCTC-3'; sgRNA-2 forward, 5'-ACCGGAAAATCTGGCCAC TCTCTT-3' and sgRNA-2 reverse, 5'-AAACAAGAGAGTG GCCAGATTTTC-3'; sgRNA-3 forward, 5'-ACCGGG CCACTCTCTTTGGAGATA-3' and sgRNA-3 reverse, 5'-AAACTATCTCCAAAGAGAGTGGCC-3'. The complementary oligonucleotides were annealed and cloned into the pGL3-U6 expression vector. To evaluate CRISPR-mediated gene knockout efficiency, MCM10 genomic fragments containing sgRNA target sites were inserted into the pmCherry$\mathrm{C} 1$-enhanced green fluorescent protein (EGFP) plasmid. The insertion of MCM10 genomic sequence created a frameshift mutation that led to disruption of the expression of EGFP. When the inserts were removed by sgRNA-guided Cas9 endonuclease, EGFP expression was detected, thus ind ting the knockout efficiency. A plasmid expressing human fas' was obtained from Addgene. Full-length $M C M 10 \mathrm{cDN}$ i pCMV6-Entry vector was obtained from O sen Jechno gies (Rockville, MD, USA).

\section{Cell transfection and rting}

EC109 cells $\left(1 \times 10^{6}\right.$ cells $\left./ 11\right)$ w seeded ono six-well plates and transfected wi the Cas9 vech sgRNA-expressing plasmid, and report plasmid $(0.8 \mu \mathrm{g}$ foreach) with Lipofectamine 3000 (T 'mo F' der Scientific). Forty-eight hours after transfection, tra ected ce' were observed under a fluoresce mio scope abjected to flow cytometric sortin ells sh in strong EGFP signals were sorted. Cells without $\mathrm{t}$-ction of tre sgRNA-expressing plasmid were used as a con 1. The sorted cells were plated at a density of 1 cell/well onto $\lambda$-well plates by limiting dilution. Cell clones were collected and tested for gene mutation or deletion.

In rescue experiments, MCM10-depleted EC109 cells were transfected with a plasmid expressing a constitutively active isoform of Akt or empty vector (Addgene, Cambridge, MA, USA) using Lipofectamine 2000. Twenty-four hours after transfection, cells were tested for proliferation and migration. For inhibitor experiments, K510 ESCC cells were pretreated with LY294002 (25 $\mu \mathrm{M}$; Sigma, St Louis, MO,
USA) or vehicle for $30 \mathrm{~min}$ at $37^{\circ} \mathrm{C}$ before transfection with MCM10-overexpressing plasmid or empty vector.

\section{T7 endonuclease I assay}

Genomic fragments containing the sgRNA-1 target site were amplified by PCR with the following primers: forward, 5'-CGTGCTTATTCTCTGTCCTTTCTC-3' and reverse, 5'-CTGGCCCAAACATTTCATCTACCA-3'. PCR products were purified and mixed with wild-type genomic DNA (in a $1: 1$ ratio). The mixture was denatured at $100^{\circ} \mathrm{C}$ for $5 \mathrm{~min}$ and annealed at room temperature. Afte with $\mathrm{T} 7$ endonuclease I (New England Biolab pswich, $\mathrm{USA}$ ) at $37^{\circ} \mathrm{C}$ for $2 \mathrm{~h}$, the resulting fragments re subjected o $1 \%$ agarose gel electrophoresis and aned wh thidiun oromide.

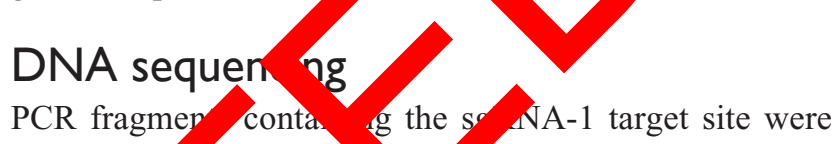
ligated to ${ }^{\prime}$ simple vec ar subjected to DNA sequencing perfumed $b$, Yhanghai Sangon Biotechnology Company (Shai, China)

\section{Cell grov h assay}

lls were $\mathrm{p}$ ) ed in 24-well plates $\left(5 \times 10^{3}\right.$ cells/well $)$ and cun foys and counted using a hemocytometer. Each xperiment with six replicates was repeated three times.

\section{Colony formation assay}

EC109 cell clones expressing wild-type and mutant MCM10 were seeded onto six-well plates (1,000 cells/well) and cultured for 3 weeks. Colonies were stained with 1\% bromophenol blue and counted. For soft-agar colony formation assay, DMEM containing $0.6 \%$ agar and $10 \% \mathrm{FBS}$ was plated on six-well plates. After solidification, cells (1,000 cells/well) suspended in culture medium containing $0.4 \%$ agar and $10 \%$ FBS were added on the gel. Cells were incubated for 3 weeks at $37^{\circ} \mathrm{C}$. Visible colonies were photographed and counted.

\section{In vitro wound-healing assay}

Cells were seeded onto six-well plates $\left(6 \times 10^{5}\right.$ cells/well $)$ and allowed to grow to $90 \%$ confluence. The cell monolayer was scratched with a $200-\mu \mathrm{L}$ pipette tip. To block cell proliferation, mitomycin-C (Sigma; $1 \mu \mathrm{g} / \mathrm{mL}$ ) was added in the media. After incubation for $48 \mathrm{~h}$, cells were photographed. Wound healing was quantified by measuring the shortest distance between scratch edges at 0 and $48 \mathrm{~h}$ after scratching.

\section{Western blot analysis}

Cell lysates were prepared in lysis buffer (50 mM Tris- $\mathrm{HCl}$ (pH 8.0), $150 \mathrm{mM} \mathrm{NaCl}, 1 \%$ NP40, 0.5\% deoxycholate, 
and $0.1 \%$ sodium dodecyl sulfate [SDS] ) containing $1 \mu \mathrm{g} / \mathrm{mL}$ aprotinin, $1 \mu \mathrm{g} / \mathrm{mL}$ leupeptin, and $1 \mathrm{mmol} / \mathrm{L}$ phenylmethylsulfonyl fluoride (Sigma). Protein concentration was measured using the Protein Assay kit (Bio-Rad, Hercules, CA, USA). Equal amounts of protein samples were separated by SDS-polyacrylamide gel electrophoresis and transferred to nitrocellulose membranes. The membranes were incubated with anti-Akt (\#9272, Cell Signaling Technology, Danvers, MA, USA; 1:500 dilution), anti-phospho-Akt (\#9271, Cell signaling; 1:300 dilution), and anti- $\beta$-actin (Santa Cruz Biotechnology, Santa Cruz, CA, USA; 1:2,000 dilution). Horseradish peroxidase-conjugated immunoglobulin G (Santa Cruz Biotechnology; 1:5,000 dilution) was used as a secondary antibody. Signals were visualized by enhanced chemiluminescence (Amersham Biosciences, Buckinghamshire, UK).

\section{Statistical analysis}

Comparison of quantitative data was determined by the Student's $t$-test, unless otherwise specified. The relationship between MCM10 expression and clinicopathologic factors was analyzed using the chi-square test. $P<0.05$ was considered statistically significant.

\section{Results}

\section{MCMIO is upregulated in ESCC}

Immunohistochemical staining for MCM10 was performed in 64 pairs of formalin-fixed, paraffin-emb SCC samples and adjacent normal esophageal thes. It wa "ound that MCM10 was predominantly deted in the cy plasm of tumor cells (Figure 1A). Loy nd high e ressior i MCM10 was detected in $27 \%(17,4)$ and $\%(4 \lambda$ of the ESCC specimens and 70\% (4. 4) $30 \%$ 19/64) of normal

A

B

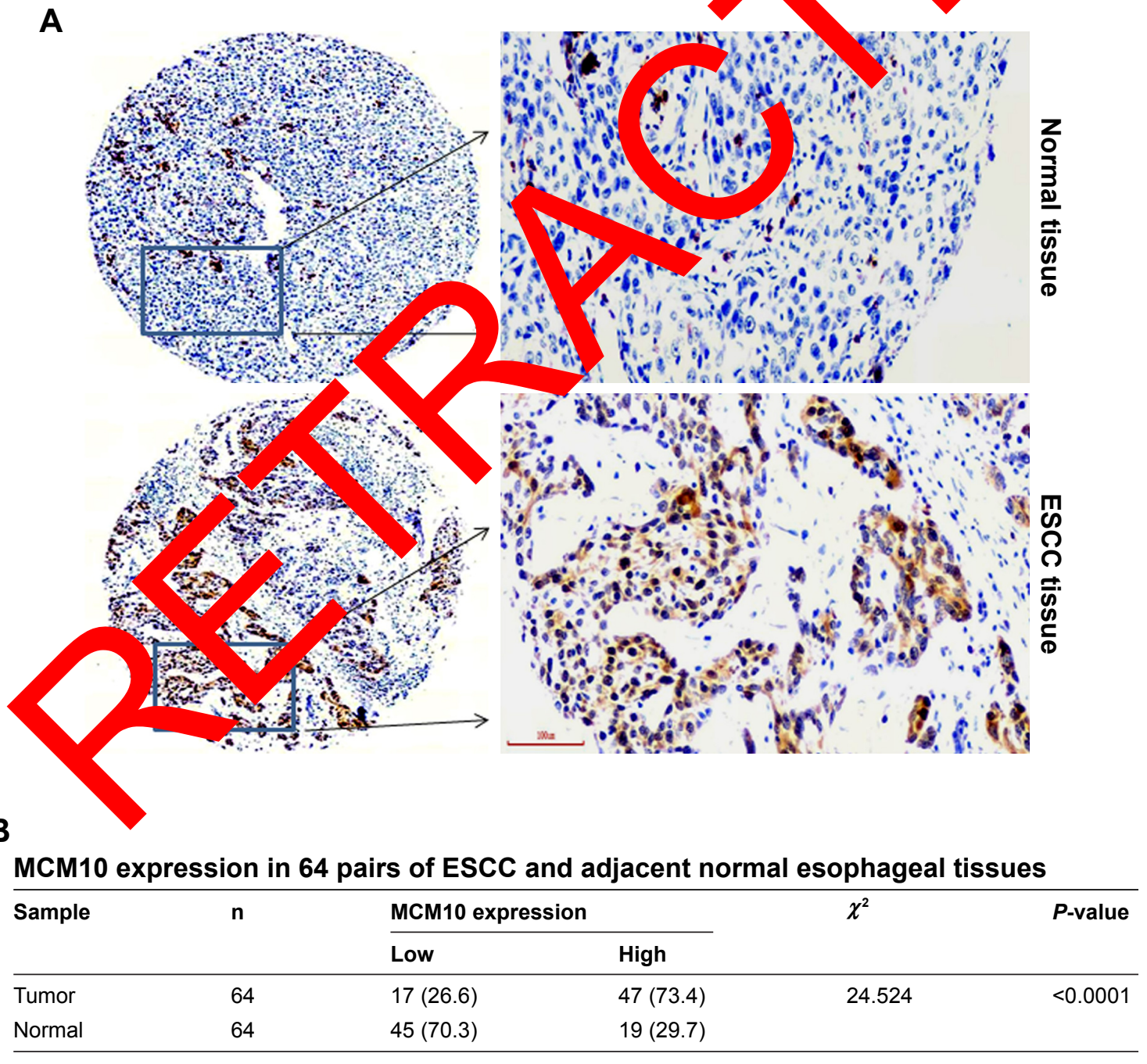

Figure I (Continued) 

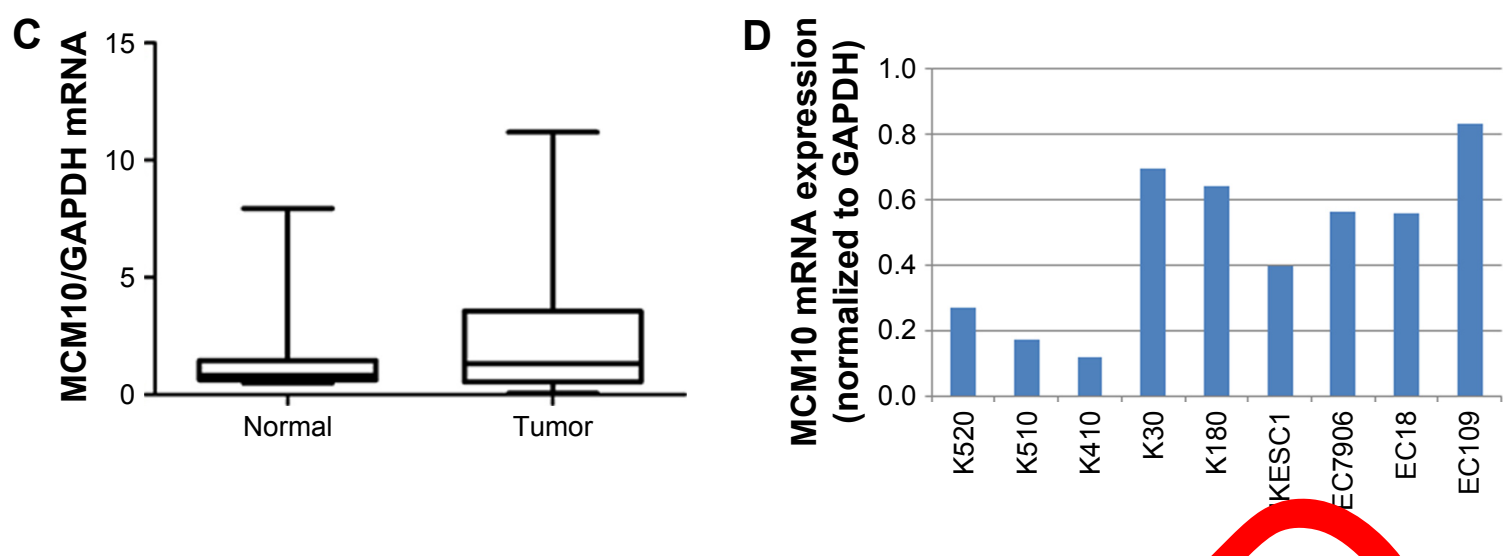

Figure I MCMIO is upregulated in ESCC.

Notes: (A) Representative images showing a pair of paraffin-embedded ESCC and adjacent normal esophageal tissues im Scale bar $=60 \mu \mathrm{m}$. (B) Summary of the immunohistochemical results in 64 pairs of paraffin-embedded ESCC and adjacent ormal hageal tissues C) Real-time PCR analysis of MCMIO mRNA levels in 56 pairs of fresh ESCC samples and corresponding normal tissues. (D) Real-time P analysis of a 10 mRD levels in a panel of ESCC cell lines.

Abbreviations: ESCC, esophageal squamous cell carcinoma; GAPDH, glyceraldehyde 3-phosphate dehydroge 2; MCMI0 inichromo, maintenance 10; PCR, polymerase chain reaction.

esophageal tissues, respectively (Figure 1B). There was a significant difference in MCM10 protein levels between ESCC and adjacent normal tissues $(P<0.0001$; Figure 1B). We also examined the mRNA expression of MCM10 in 56 pairs of fresh ESCC samples and corresponding normal tissues by real-time PCR analysis. The results showed the the amounts of MCM10 transcripts were significantly gre er ESCC than in normal tissues $(P<0.001$; Figure 1C). Comp ed to the other ESCC cell lines tested, K520 cells showed lower levels of MCM10 $\mathrm{NA}$ (F) ure 1D)

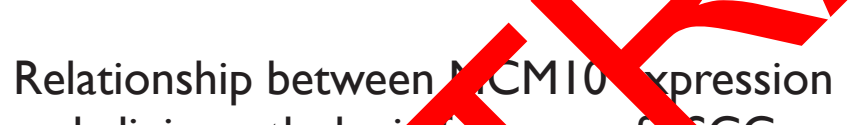
and clinicopathologi te. vres of SCC

Next, we assessed the co elations bet en MCM10 expression and clinicopath gic features of ESO . As summarized in Table 1, the ressig of MCM10 was significantly associated with age agnosis $=0.033$ ). However, no significan uffer ce wa lete detween MCM10 levels and ot param ers including gender, differentiation grade, invasion , and Trem stage.

\section{Deletion o.MCMI0 through CRISPR/} Cas9 technology

EC109 cells were transfected with the Cas9, sgRNAs, and reporter constructs, and after incubation for $48 \mathrm{~h}$, the reporter expression was examined. The expression of EGFP was found in some reporter-transfected clones, indicating the removal of the MCM10 sgRNA target site in the reporter by sgRNA-guided Cas9 (Figure 2A). Moreover, delivery of
sgRNA fielo the highes, nockout efficiency, compared to the other two SNAs (data not shown). Therefore, in following experin. nts, we used sgRNA-1 to eliminate ICM10 exp ssion. Flow cytometric sorting showed that $\%$ of sgRN -1-transfected cells emitted both red and green flu. (data not shown). Flow cytometry sorted cells were expanded to form single-cell clones. PCR amplicons Werwsubjected to the mismatch-sensitive $\mathrm{T} 7$ endonuclease assay (Figure 2B). It was found that out of the 96 cell clones tested, 72 had mutations induced by sgRNA-guided Cas9. Sequencing of the PCR products demonstrated that 30 of the

Table I Relationship between MCMIO expression and clinicopathologic features of ESCC $(n=64)$

\begin{tabular}{|c|c|c|c|}
\hline \multirow[t]{2}{*}{ Variable } & \multicolumn{2}{|c|}{ MCMIO expression } & \multirow[t]{2}{*}{$P$-value } \\
\hline & Low & High & \\
\hline \multicolumn{3}{|l|}{ Gender } & 0.635 \\
\hline Male & 8 & 19 & \\
\hline Female & 9 & 28 & \\
\hline \multicolumn{3}{|l|}{ Age (years) } & 0.033 \\
\hline$<60$ & 14 & 19 & \\
\hline$\geq 60$ & 5 & 28 & \\
\hline \multicolumn{3}{|l|}{ Differentiation } & 0.564 \\
\hline Advanced & 3 & 12 & \\
\hline Moderate & 11 & 32 & \\
\hline Poor & 3 & 3 & \\
\hline \multicolumn{3}{|c|}{ Tumor-node-metastasis stage } & 0.777 \\
\hline $\mathrm{I} / \mathrm{II}$ & 8 & 24 & \\
\hline III/IV & 9 & 23 & \\
\hline \multicolumn{3}{|c|}{ Lymph-node metastasis } & 0.602 \\
\hline Absent & II & 27 & \\
\hline Present & 6 & 20 & \\
\hline
\end{tabular}

Abbreviations: ESCC, esophageal squamous cell carcinoma; MCMI0, minichromosome maintenance 10. 
A
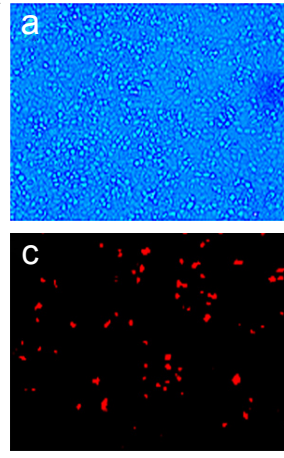

C

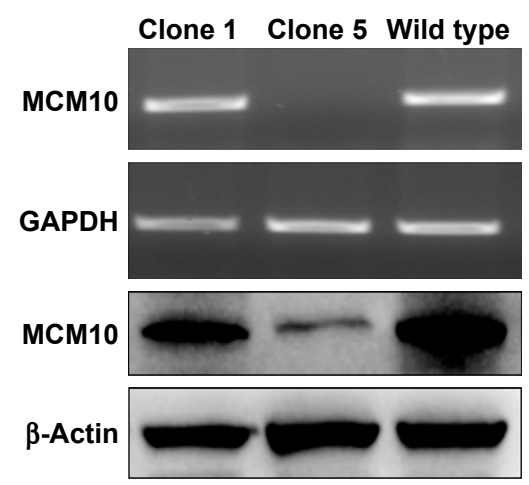

B
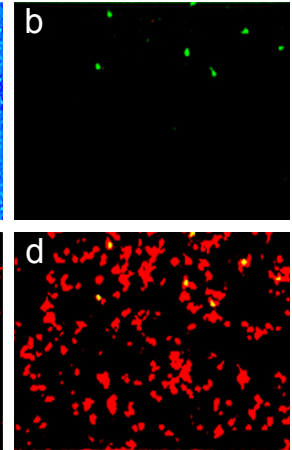

D

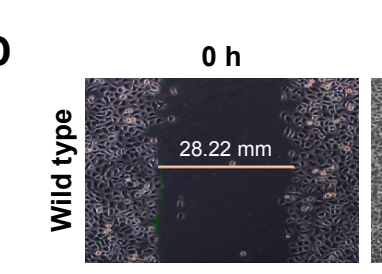

MCM10-targeted cell clones
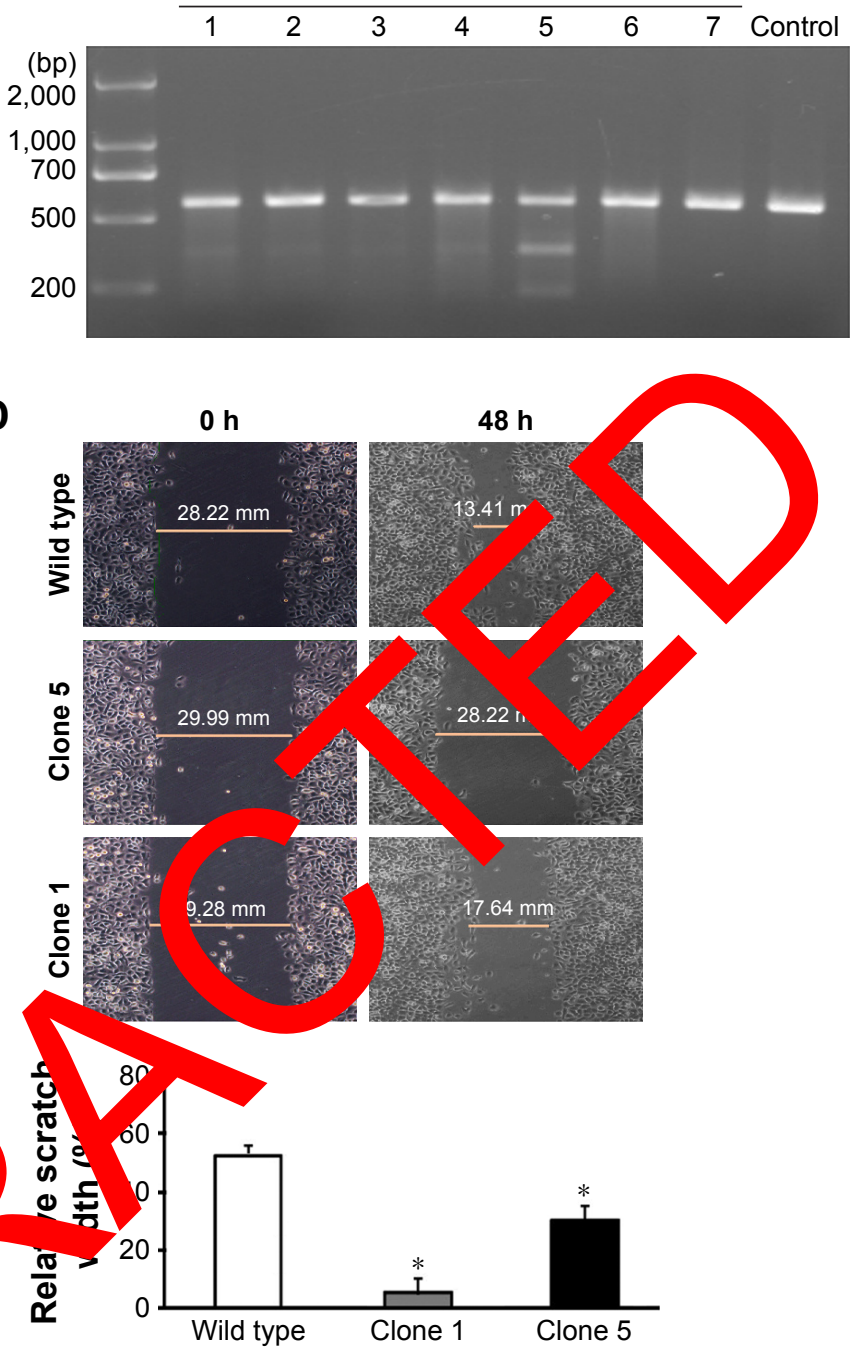

Figure 2 Deletion of MCMI0 through CPISPR/Cas9 tec,

Notes: (A) Validation of CRISPR/C mediated knockou "iciency using a mCherry/GFP reporter construct. ECI09 cells were transfected with the Cas9, sgRNAs, and reporter constructs, and mC y/GFP fluorescence was amined $48 \mathrm{~h}$ after transfection. (a) Bright field image of cells. (b) Some cells displayed GFP fluorescence, indicating the presence of CRIS Cas9-med removal of target sequence. (c) ECI09 cells that were transfected with reporter construct showed mCherry fluorescence. (d) Merged image of green and fluor ence yielded yellow fluorescence. Scale bar $=100 \mu \mathrm{m}$. (B) T7 endonuclease assay. Different clones derived from ECI09 cells transfected with Cas9 and sgRNA subjected CR amplification of genomic DNA containing sgRNA-I target site. The size of T7 endonuclease I-digested DNA fragments is indicated of co l, negatrol. (C) Upper; RT-PCR analysis of MCMI0 mRNA expression in different ECI09 sublines. Lower; Western blot analysis of MCMIf otein le Is. (D) D f MCMIO hampers the migration of ESCC cells. In vitro wound-healing assay was performed to assess cell migration capacity. Top; $f$ represent e experimen, he percentage of wound closure was determined from three independent experiments. $* P<0.05$ vs wild-type cells.

Abbreviation CC, es $\quad$, maintenance $10 ; \mathrm{R}$,everse transcription polymerase chain reaction.

33 samples $(90.9 \%)$ had deletion mutations, two $(6 \%)$ frameshift mutations, and one wild-type. Additionally, RT-PCR and Western blot analyses confirmed the ablation of MCM10 in the Clone 5 subline (Figure 2C).

\section{Depletion of MCMIO suppresses the migration of ESCC cells}

We also checked the effect of MCM10 knockout on the migration of ESCC cells. The percentage of wound closure was markedly reduced in the Clone 5 subline compared to that in the Clone 1 subline (Figure 2D). Moreover, the Clone 1 subline exhibited a reduced migration capacity relative to wild-type EC109 cells (Figure 2D). These observations suggest an important role for MCM10 in the regulation of ESCC cell migration.

\section{MCMIO is required for the growth of ESCC cells}

Next, we assessed the impact of MCM10 deficiency on the growth of EC109 cells. We found that the Clone 5 
A

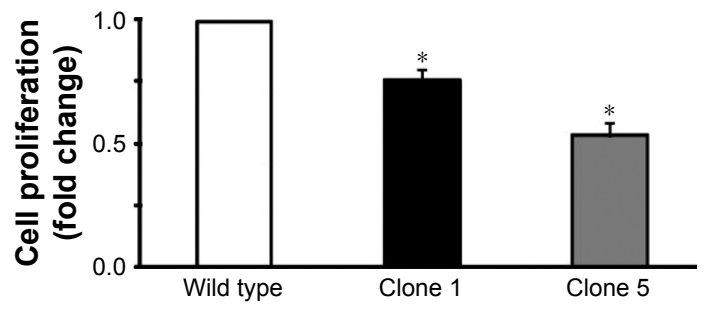

B

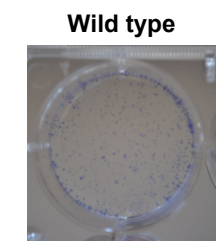

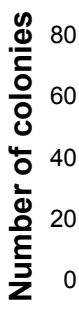

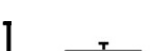

Clone 1
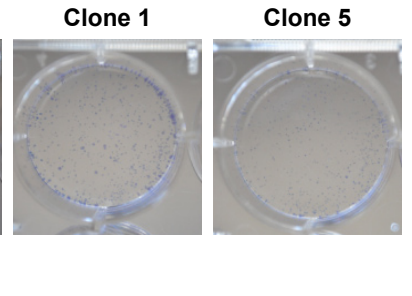

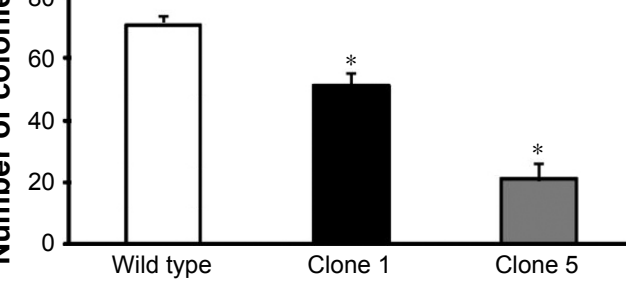

Figure 3 MCMIO is required for the growth of ESCC cells.

Notes: (A) Cells were cultured for 7 days and counted using a hemocytometer. Each low density and allowed to form colonies after culturing for 3 weeks. (C) Soft-agar cc plating. $* P<0.05$ vs wild-type cells.

Abbreviations: ESCC, esophageal squamous cell carcinoma; MCMI0, minichromosome

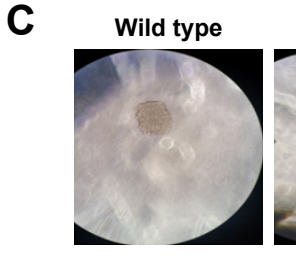

Clone 1

Clone 5
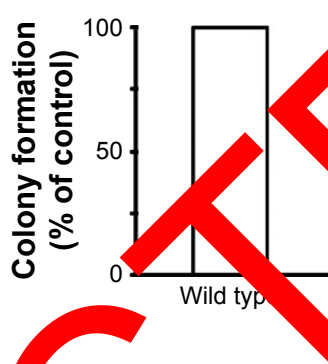

periment with simreplicates was repeated three times. (B) Cells were plated at a vy formation ass The number of colonies formed was quantified 3 weeks after tenance subline had a significantly lower proliferation cap it than the Clone 1 subline $(P<0.05$; Figu Col formation assay confirmed that the clone sublin formed significantly fewer colonies line $(P<0.05$; Figure 3B). To iny gate th se of MCM10 in the anchorage-independen of of ESO cells, softagar colony formation assay was po rmed. As depicted in Figure 3C, the numbe of colonies fon d by the Clone 5 subline was signif antly lo er than that by the Clone 1 subline $(P<0.05)$. ese sults indicate that MCM10 plays an essential in the owth o $\angle$ SCC cells.

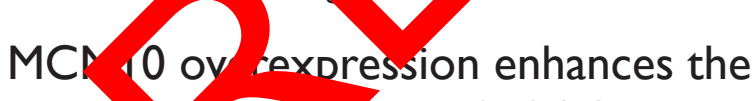
growt a migration of ESCC cells

Next, we exp ad the effect of ectopic expression of MCM10 on the aggressive phenotype of ESCC cells. It was found that overexpression of MCM10 (Figure 4A) significantly promoted the growth (Figure 4B) and migration (Figure 4C) of both K510 and K520 ESCC cells.

\section{MCMIO loss-induced suppression of growth and migration involves inhibition of Akt signaling}

Western blot analysis revealed that MCM10 depletion led to a marked inhibition of Akt phosphorylation on Ser473 1iv 109 cells (Figure 5A). However, the phosphorylation levels of nuclear factor kappa B (NF-кB), extracellular signal-regulated kinase (ERK), and p38 protein were not altered by MCM10 depletion (data not shown). To confirm the role of Akt signaling in the action of MCM10, we performed rescue experiments in the Clone 5 subline using constitutively active Akt (Figure 5B). Of note, overexpression of constitutively active Akt restored cell growth (Figure 5C) and migration (Figure 5D) in MCM10-deficient cells. In contrast, ectopic expression of active Akt had no significant impact on the proliferation of wild-type EC109 cells. In addition, the migration was moderately increased by overexpression of active Akt in wild-type EC109 cells.

\section{Inhibition of Akt signaling blocks MCMIO- induced cell proliferation and migration}

Western blot analysis indicated that overexpression of MCM10 promoted Akt phosphorylation in K520 cells transfected with MCM10-expressing plasmid (Figure 6A). We pretreated K520 cells with LY294002 or vehicle before transfection with empty vector or MCM10-expressing plasmid. Inhibition of Akt phosphorylation can restrain cell growth (Figure 6B) and cell migration capacity (Figure 6C) in MCM10-overexpressed cells. 


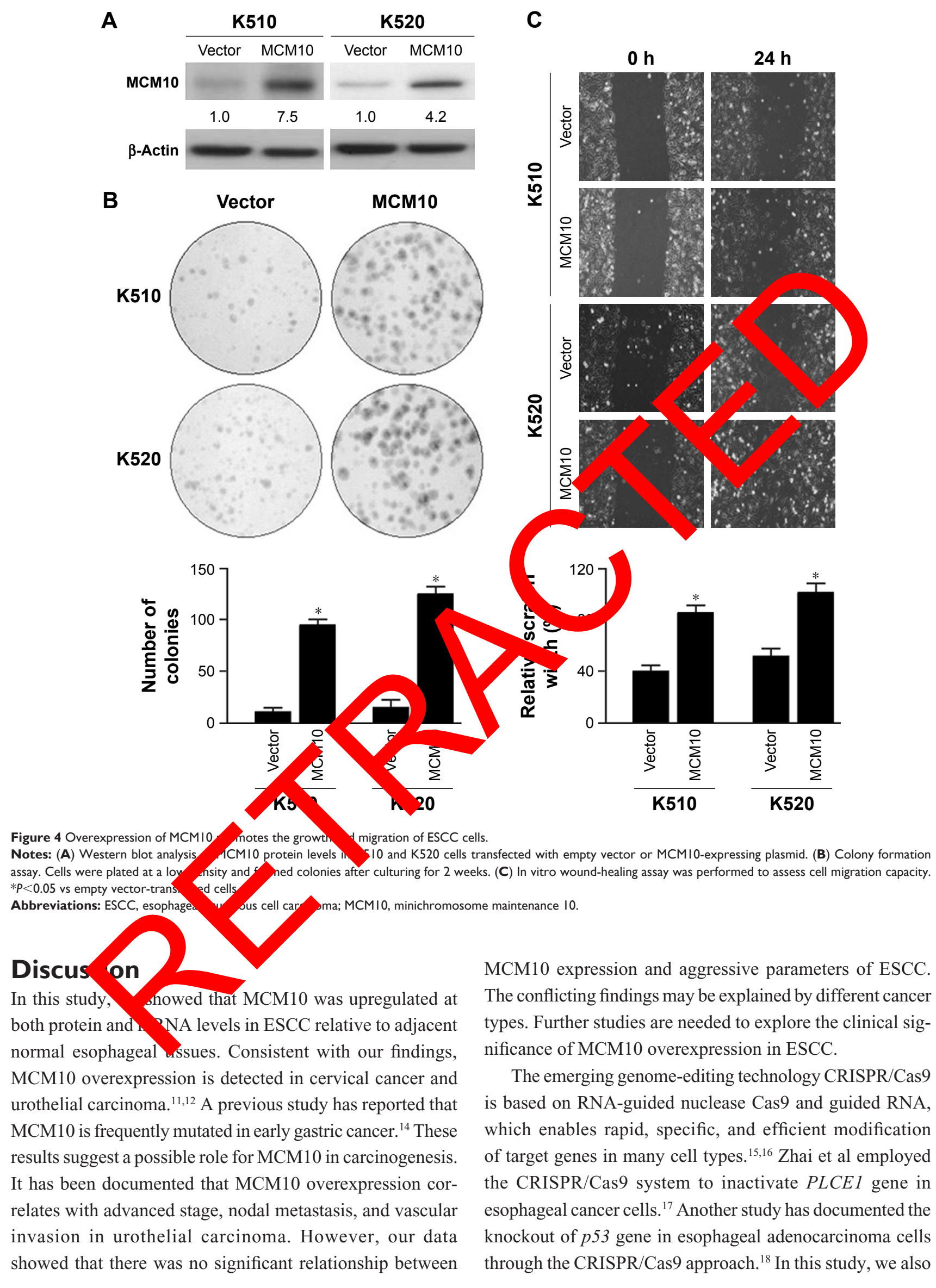


A

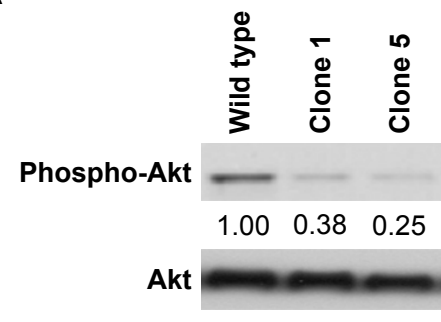

B

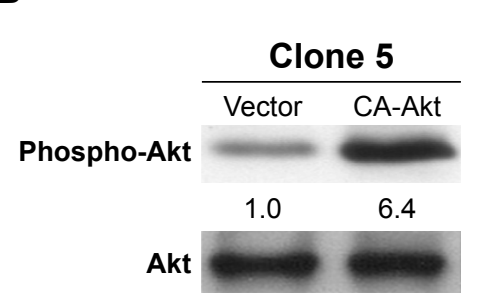

C

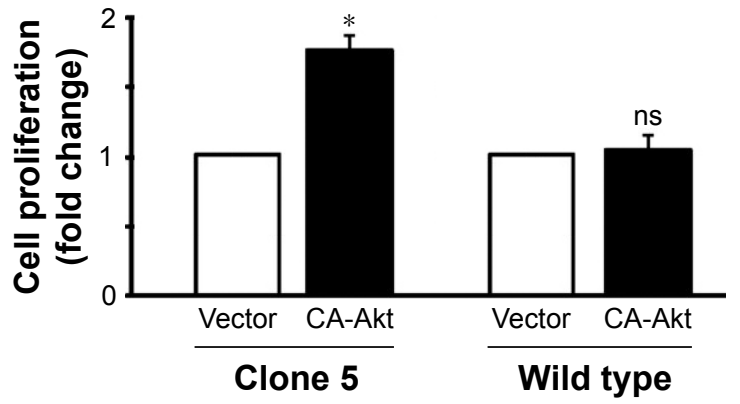

D

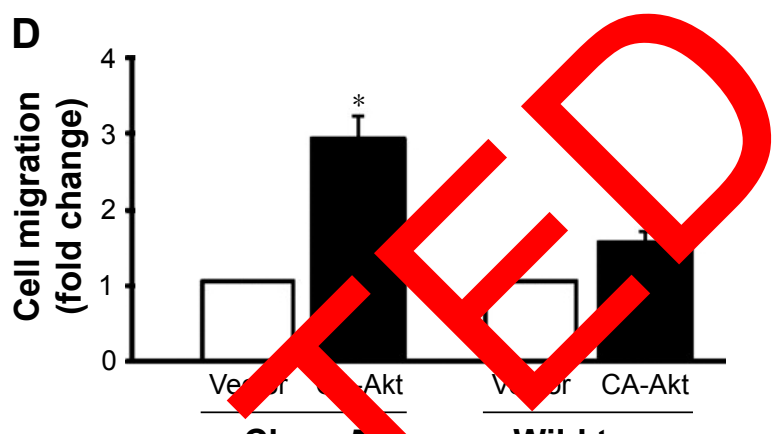

Figure 5 MCMIO loss-induced suppression of growth and migration involves inhibition Notes: (A) Western blot analysis of Akt phosphorylation in different ECI09 sublines. with constitutively active Akt or empty vector. Numbers indicate fold change relative to cell growth $(\mathbf{C})$ and migration $(\mathbf{D})$ in the Clone 5 subline. ${ }^{*} \mathrm{P}<0.05$ vs vector control.

Clone 5

Wild type Abbreviations: MCMI0, minichromosome maintenance I0; ns, no statistical significance.

Akt signaling.

Western blot ysis of Akt phosphorylation in the Clone 5 subline transfected wild-type group $\quad \mathbf{C}$ and $\mathbf{D}$ ) Overexpression of constitutively active Akt restored

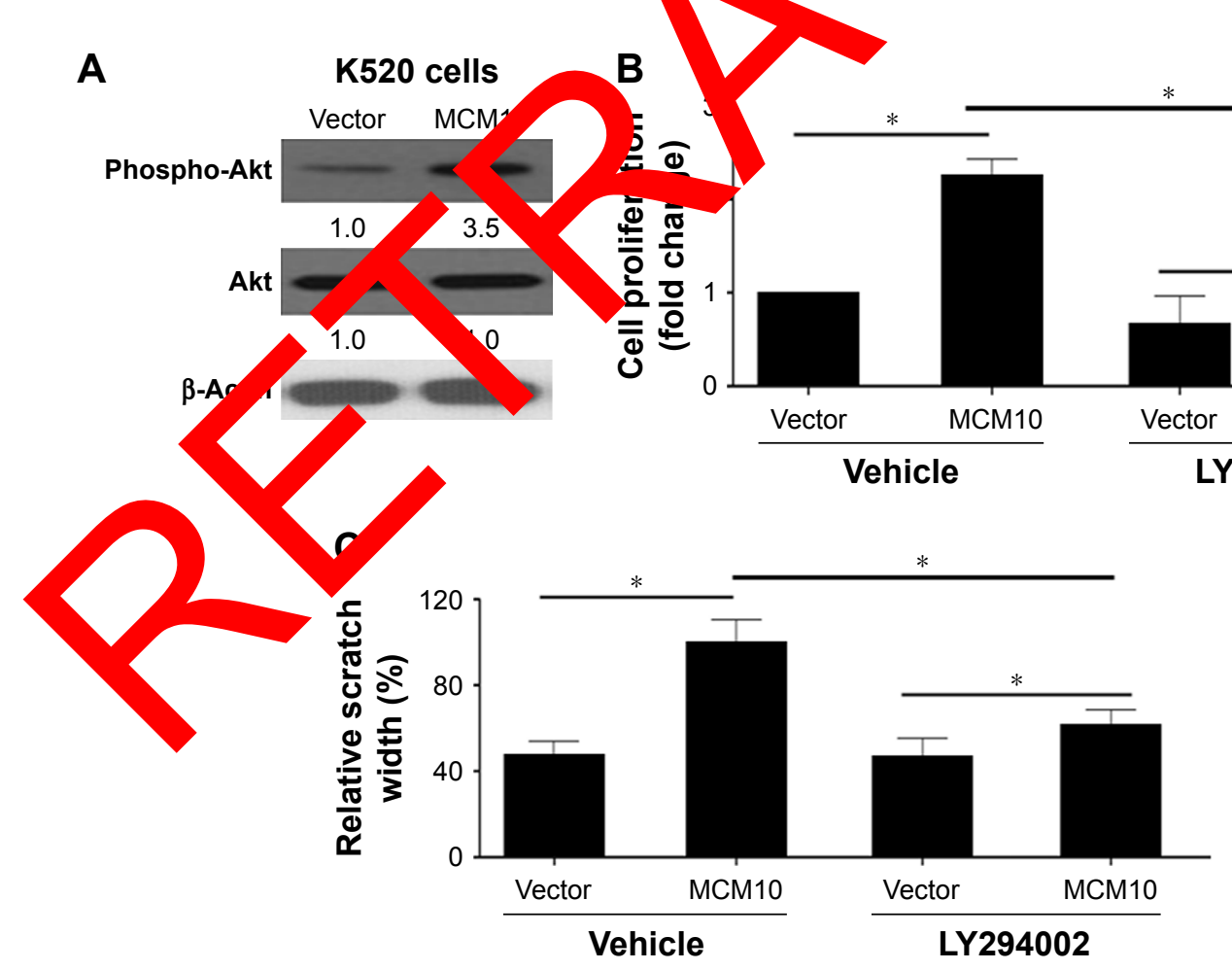

Figure 6 Inhibition of Akt signaling blocks MCMI0-induced cell proliferation and migration.

Notes: (A) Western blot analysis of Akt phosphorylation in K520 cells transfected with empty vector or MCMI0-expressing plasmid. (B) K520 cells were pretreated with LY294002 or vehicle before transfection with empty vector or MCMI0-expressing plasmid. Cells were cultured for 7 days and counted using a hemocytometer. Each experiment with six replicates was repeated three times. (C) In vitro wound-healing assay was performed to assess cell migration capacity. $* P<0.05$ vs empty vector-transfected cells. Abbreviations: MCMI0, minichromosome maintenance 10; ns, no statistical significance. 
successfully inactivated MCM10 in EC109 cells through the CRISPR/Cas9 technology. Of note, knockout of MCM10 significantly impaired the growth and colony formation of EC109 cells. Moreover, MCM10 knockout suppressed the anchorage-independent growth of EC109 cells on soft agar. In addition, the migration capacity was reduced in MCM10null EC109 cells relative to wild-type equivalents. These results collectively indicate that $\mathrm{MCM} 10$ is required for the maintenance of the aggressive phenotype in ESCC cells.

MCM10 is implicated in MCM2-7 remodeling and cellcycle progression and its functional deletion causes $S$ phase defects, ${ }^{19}$ which may explain the reduced growth in MCM10-null EC109 cells. In addition to regulation of cell-cycle progression, MCM proteins have the capacity to modulate cell migration and invasion. It has been documented that overexpression of MCM2, MCM3, and MCM7 facilitates cell migration and invasion in medulloblastoma cells. ${ }^{20}$ Another study showed that MCM7 knockdown hinders the migration of KYSE510 and EC9706 cells in vitro. ${ }^{21}$ In this study, we provide evidence for the importance of MCM10 in the migration of ESCC cells. Therefore, the interaction between MCM10 and MCM2-7 proteins may be critical for the growth and progression of ESCC.

To get more insight into the action of MCM10, examined the signaling pathways involved. We noted tha MCM10 null suppressed the phosphorylation spared NF-кB, ERK, and p38 in EC109 ce', sugg ting the involvement of Akt signaling in the act

Rescue experiments provided direct denco averexpression of constitutively active $A$ celerated $t$ growth and migration of MCM10-null_C109 1ls. Activaton of the Akt pathway is implice in the grow and migration of ESCC cells induce oy maty metalloproteinase-1.22 Another study demons, ed therexpression of inhibitor of differentiation $\mathrm{DN}$, inding omotes growth and invasion of ophag 1 canc $1 \mathrm{~S}$ through the Akt signaling pat ay. ${ }^{23} \mathrm{~T}$ atudies, combined with our data, point toward mportance of Akt signaling in the action of MCM10 in Es C. However, further studies are needed to clarify the mecharism by which MCM10 regulates the activation of Akt signaling.

A major limitation of this study is that the role of MCM10 was tested in only one ESCC cell line. Ongoing studies are designed to address the effect of targeted ablation of MCM10 on tumor growth and metastasis in mouse models inoculated with multiple ESCC cell lines.

In conclusion, our data demonstrate that MCM10 is upregulated in ESCC and contributes to the aggressive phenotype of ESCC cells. Activation of Akt signaling is involved in the oncogenic activity of MCM10 in ESCC. Targeting MCM10 may provide a potential therapeutic benefit in the treatment of ESCC.

\section{Data sharing statement}

All the data obtained from the present study are available from the corresponding author under reasonable request.

\section{Acknowledgments}

This work was funded under the Youth Fund of First Affiliated Hospital of Zhengzhou Unive ity. Th uthors are grateful to all study participants.

\section{Disclosure}

The authors report no

\section{Reference}

1. Torre LA, B A, rel RL, Ferlay tet-Tieulent J, Jemal A. Global cancer statistics, 201 CA Cancer J Clin. 2015;65(2):87-108.

2. Zhano $D$ L iXM, Zhao $X$ al. Novel genetic locus at MHC region for es ragearsquamous cell ca noma in Chinese populations. PLoS One. 17;12(5):e0177494.

3. n Hagen P, H hof MC, van Lanschot JJ, et al; CROSS Group. operative che radiotherapy for esophageal or junctional cancer. M. $\quad$ J Med.J 2;366(22):2074-2084.

4. Best L_ al M, Gurusamy KS. Non-surgical versus surgical treatant for oesophageal cancer. Cochrane Database Syst Rev. 2016;3: CD. 8 .

Ganaie SS, Zou W, Xu P, Deng X, Kleiboeker S, Qiu J. Phosphorylated STAT5 directly facilitates parvovirus B19 DNA replication in human erythroid progenitors through interaction with the MCM complex. PLoS Pathog. 2017;13(5):e1006370.

6. Maiorano D, Lutzmann M, Méchali M. MCM proteins and DNA replication. Curr Opin Cell Biol. 2006;18(2):130-136.

7. Lõoke M, Maloney MF, Bell SP. MCM10 regulates DNA replication elongation by stimulating the CMG replicative helicase. Genes Dev. 2017;31(3):291-305.

8. Stewart PA, Khamis ZI, Zhau HE, et al. Upregulation of minichromosome maintenance complex component 3 during epithelial-to-mesenchymal transition in human prostate cancer. Oncotarget. 2017;8(24):39209-39217.

9. Coli A, Asa SL, Fadda G, et al. Minichromosome maintenance protein 7 as prognostic marker of tumor aggressiveness in pituitary adenoma patients. Eur J Endocrinol. 2016;174(3):307-314.

10. Abe S, Yamamoto K, Kurata M, et al. Targeting MCM2 function as a novel strategy for the treatment of highly malignant breast tumors. Oncotarget. 2015;6(33):34892-34909.

11. Das M, Prasad SB, Yadav SS, et al. Over expression of minichromosome maintenance genes is clinically correlated to cervical carcinogenesis. PLoS One. 2013;8(7):e69607.

12. Li WM, Huang CN, Ke HL, et al. MCM10 overexpression implicates adverse prognosis in urothelial carcinoma. Oncotarget. 2016;7(47): 77777-77792.

13. Schmittgen TD, Livak KJ. Analyzing real-time PCR data by the comparative C(T) method. Nat Protoc. 2008;3:1101-1108.

14. Kang G, Hwang WC, Do IG, et al. Exome sequencing identifies early gastric carcinoma as an early stage of advanced gastric cancer. PLoS One. 2013;8(12):e82770.

15. Chira S, Gulei D, Hajitou A, Zimta AA, Cordelier P, Berindan-Neagoe I. CRISPR/Cas9: transcending the reality of genome editing. Mol Ther Nucleic Acids. 2017;7:211-222. 
16. Zhu P, Wu F, Mosenson J, Zhang H, He TC, Wu WS. CRISPR/Cas9mediated genome editing corrects dystrophin mutation in skeletal muscle stem cells in a mouse model of muscle dystrophy. Mol Ther Nucleic Acids. 2017;7:31-41.

17. Zhai S, Liu C, Zhang L, et al. PLCE1 promotes esophageal cancer cell progression by maintaining the transcriptional activity of snail. Neoplasia. 2017;19(3):154-164.

18. Liu DS, Read M, Cullinane C, et al. APR-246 potently inhibits tumour growth and overcomes chemoresistance in preclinical models of oesophageal adenocarcinoma. Gut. 2015;64(10):1506-1516.

19. Quan Y, Xia Y, Liu L, et al. Cell-cycle-regulated interaction between MCM10 and double hexameric Mcm2-7 is required for helicase splitting and activation during S Phase. Cell Rep. 2015;13(11): 2576-2586.
20. Lau KM, Chan QK, Pang JC, et al. Minichromosome maintenance proteins 2, 3 and 7 in medulloblastoma: overexpression and involvement in regulation of cell migration and invasion. Oncogene. 2010;29(40): 5475-5489.

21. Qiu YT, Wang WJ, Zhang B, Mei LL, Shi ZZ. MCM7 amplification and overexpression promote cell proliferation, colony formation and migration in esophageal squamous cell carcinoma by activating the AKT1/ mTOR signaling pathway. Oncol Rep. 2017;37(6):3590-3596.

22. Liu M, Hu Y, Zhang MF, et al. MMP1 promotes tumor growth and metastasis in esophageal squamous cell carcinoma. Cancer Lett. 2016 377(1):97-104.

23. Li B, Tsao SW, Li YY, et al. Id-1 promotes tumorigenicity and metastasis of human esophageal cancer cells through activation of PI3K/ AKT signaling pathway. Int J Cancer. 2009;125(11):2576-2585.

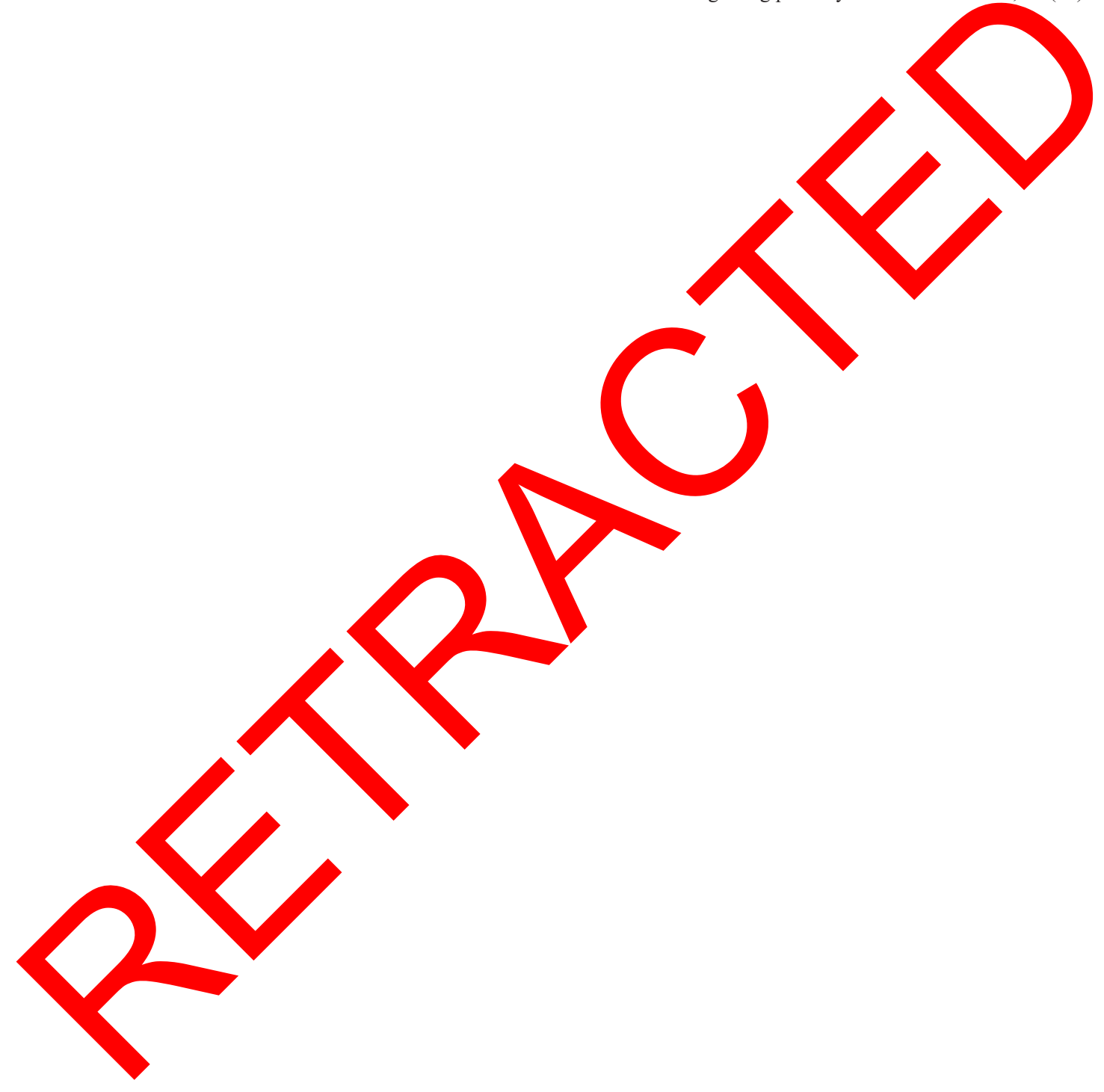

OncoTargets and Therapy

\section{Publish your work in this journal}

OncoTargets and Therapy is an international, peer-reviewed, open access journal focusing on the pathological basis of all cancers, potential targets for therapy and treatment protocols employed to improve the management of cancer patients. The journal also focuses on the impact of management programs and new therapeutic agents and protocols on

\section{Dovepress}

patient perspectives such as quality of life, adherence and satisfaction. The manuscript management system is completely online and includes a very quick and fair peer-review system, which is all easy to use. Visit http://www.dovepress.com/testimonials.php to read real quotes from published authors. 